\section{A mild pathogen turned ugly: Zika virus and the case with microcephaly}

Zika virus (ZIKV) is a mosquito-bourne, dengue-like flavivirus (arbovirus), which has been causing transient outbreaks of mild, self-limited illness for about 70 years. Its recent notoriety has arisen from its association with Guillain-Barré syndrome in the general population and outbreak of babies with microcephaly in Brazil, particularly in the northeast region after September 2015 [1, 2].

Historically, ZIKV was first isolated in 1947-1948 in the namesake forest, a small (less than 1 square mile) isolated lakeshore forest in Uganda, from a sentinel rhesus monkey and a mosquito (Aedes [Stegomyia] Africanus) [3, 4]. In a 1964 field research paper studying the way of ZIKV transmission in its native source, it was concluded that infected mosquitos could be widely spread beyond the forest by convection currents above the treetops in the few hours following sunset [4]. After the original detection in A.(S.) Africanus, ZIKV has also been isolated in many other mosquito species, including A. Luteocephalus A. Aegypti, A. Albopictus, A. Apicoargenteus, A. Vitattus, A. Hensilli and A. Furcifer [5-10].

The first cases of human ZIKV infection were reported in the 1950s and 1960s [11, 12]; since then, Zika infection has been described as a mild and transient illness, its most common symptoms and signs including rash, fever, arthritis/arthralgia, conjunctivitis, myalgia, headache, retroorbital pain, edema and vomiting [13].

Zika infection outside Africa was first reported in 1969 in Malaysia [14], and later in Indonesia [5], Pakistan [15] and a large outbreak in Oceania $[13,16]$. We currently know that there are 2 main ZIKV lineages, an African and an Asian, and the strain that caused the Micronesia (Oceania) epidemic most likely originated in Southeast Asia [17]. At that time, a prophetic paper in Emerging Infectious Diseases highlighted the potential for transmission in Pacific Ocean and the Americas [10]. This indeed happened in French Polynesia, New Caledonia, Cook Islands and Easter Island by 2014 [18], and then from Cook Islands to Australia [19]. The first registered cases in the Americas were reported in 2014, in a woman who returned in Canada from Thailand [20] and then in Texas, in a woman who returned from vacation in BoraBora [21]. Shortly after, 2 cases were reported in Italy, in patients coming from French Polynesia [22].

Sporadic reports were published until the end of 2015, when several cases of congenital microcephaly were reported in Brazil, in association with a Zika outbreak [23, 24]. The first recorded cases in Brazil were reported in June 2015, originally in an area endemic for Dengue fever [25] and soon several reports followed, including cases from Venezuela [26] and Colombia [27].

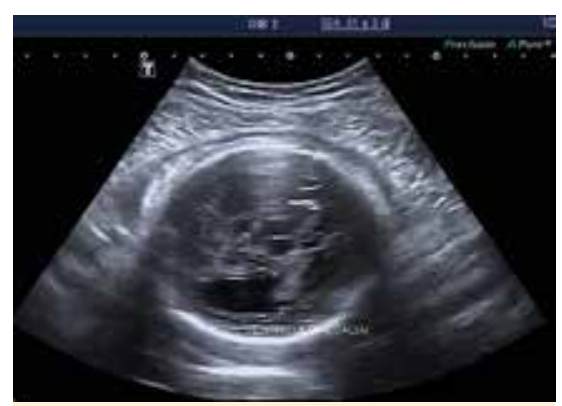

Fig. 1 Microcephaly and ventriculomegaly in a 31-weeks ZIKV affected fetus in Brazil.

Sonographic findings in the affected fetuses include microcephaly (more than 2.5 SD below mean, calcifications of the white matter, callosal and vermian dysgenesis, ventriculomegaly, thalamic hypoplasia and even arthrogryposis [28]) (• Fig. 1 and 2). Approximately 1 third of affected neonates have ocular findings, including macular alterations and optic nerve abnormalities, usually bilateral $[29,30]$.

Given that ZIKV had not been previously associated with fetal congenital defects, a crucial debate has been whether this relationship is truly causal or just statistical. The other 2 hot topics are whether ZIKV infection can be transmitted in ways other than mosquito bite (e.g. through sexual contact) and what are the potential protective strategies.

Regarding the first issue, the potential for vertical infection of ZIKV had already been described in 2 neonates in 2014; one of them was asymptomatic whereas the other developed a mild transient disease with fever and rash [31]. If ZIKV can be transmitted vertically, then the involvement of the central nervous system should not come as a complete surprise; the tropism of Zika for CNS had already been described in the first reports of Dick [3, 32], and experimental intracerebral inoculation of ZIKV in newborn mice resulted in replication of the virus in neurons and astroglial cells and eventually in their destruction, possibly though an immunological mechanism (at least partially) [33]. Although it is still not precisely known how ZIKV infection can damage the fetal brain, the paradigm of skin cells shows that the virus can infect immature dendritic cells (among others) and induce the formation of autophagosomes [34]. A more direct proof of causal relationship was provided in a recent autopsy report of a fetus with microcephaly and calcifications, in which Zika particles and RNA in were found in its brain, but no other organs [35]. However, confirmatory examinations are not the rule in ZIKV-attributed microcephaly and there are still publications highlighting the potential role of confounders (see at the end of this document).

As for potential alternative ZIKV infection routes, there is evidence for transmission via direct contact already since 2011; an American scientist who had contracted Zika when working in Senegal transmitted the virus to his wife after his return, probably through sexual contact [36].Zika virus has been isolated from the human semen [37], saliva [18] and urine [38].

For as long as there is no vaccine, the only reasonable prevention measure recommended is through avoidance of mosquito bites [39]. In its interim guideline, the US Centers for Disease Control and Prevention $(C D C)$ recommended testing of women who have travelled to endemic areas and have either developed symptoms or had ultrasound findings (microcephaly, endocranial calcifications) suggestive of fetal infection [40]. An update of the CDC guideline introduced the recommendation of preventive testing in women who have travelled in endemic areas, 2 to 12 weeks after their return from travel [41].

One of our current knowledge gaps refers to the effect of gestational age at infection and the exact magnitude of the risk. There 


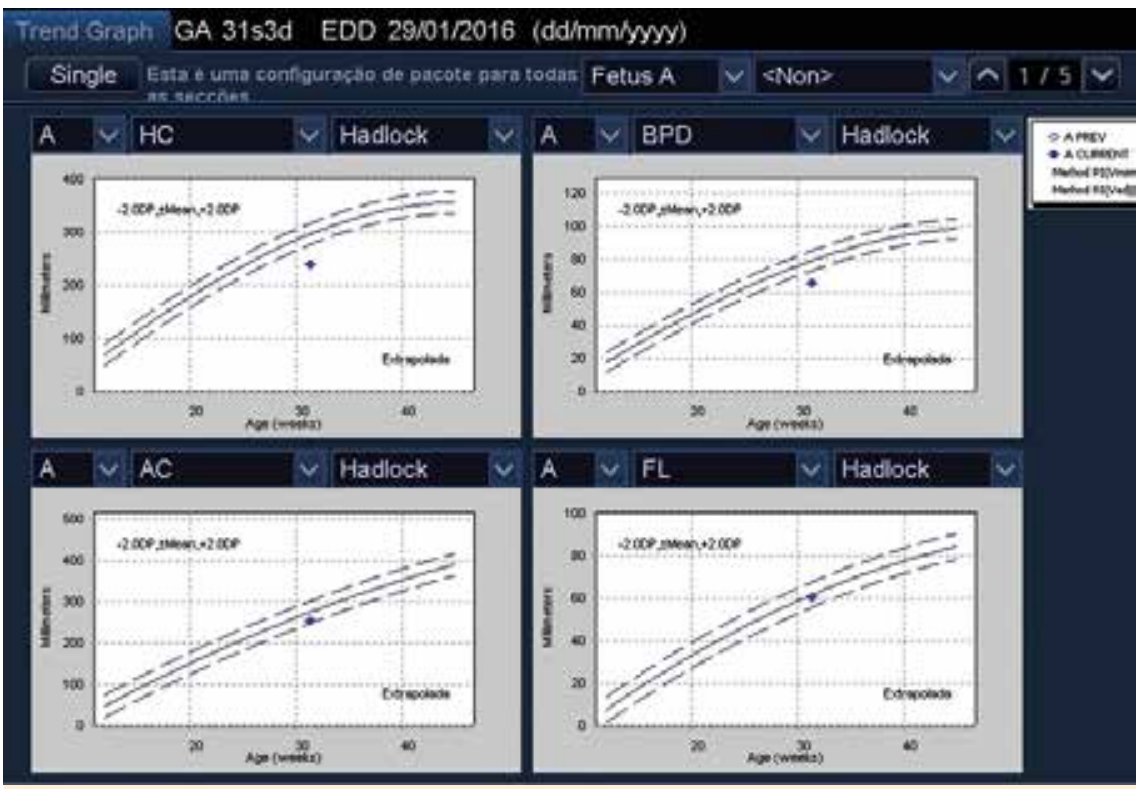

Fig. 2 Biometric charts of the same fetus, illustrating the severe lag of head measurements (BPD: biparietal diameter; HC: head circumference), which is setting the diagnosis of microcephaly. The abdominal circumference (AC) shows a slighter delay; intrauterine growth restriction has also been described in ZIKV-affected fetuses. FL: femur length

is also no proof that mosquito control measures will succeed in controlling Zika, in the same way they did not succeed in controlling the -related in phylogenetic and epidemiological terms- Dengue virus [42].
The good news is that there is significant likelihood that a ZIKV vaccine could be produced in large quantities by the end of 2016 [43]. Until then, the Zika virus outbreak is regarded as a global public health emergency by the WHO [44], which has moreover issued a warning alert for Euro- pean countries to control their populations of mosquitos of the Aedes genus in view of their expected activation during spring and summer [45].

Other interesting -and challenging-readings:

http://www.telegraph.co.uk/news/

worldnews/zika/12157747/Zika-virus-

Brazil-dismisses-link-between-larvicideand-microcephaly.html http://www.cdc.gov/mmwr/volumes/65/ $\mathrm{wr} / \mathrm{mm} 6503 \mathrm{e} 2 . \mathrm{htm}$

Alexandros Sotiriadis ${ }^{1}$, Wellington P. Martins $^{2}$, Jailson Costa Lima ${ }^{3}$, Kimon Chatzistamatiou $^{1}$

${ }^{1}$ Second Department of Obstetrics and Gynecology, Aristotle University of Thessaloniki, Thessaloniki, Greece

${ }^{2}$ University of Sao Paulo, Department of Obstetrics and Gynecology, Ribeirao Preto Medical School (FMRP-USP), Ribeirao Preto, Brazil

${ }^{3}$ Faculty of Medical Sciences - State University of Piaui (FACIME-UESPI), Brazil

References online 